\title{
Correction to: Prenatal Exposure of Cypermethrin Induces Similar Alterations in Xenobiotic-Metabolizing Cytochrome P450s and Rate-Limiting Enzymes of Neurotransmitter Synthesis in Brain Regions of Rat Offsprings During Postnatal Development
}

\begin{abstract}
Anshuman Singh ${ }^{1,2} \cdot$ Anubha Mudawal ${ }^{1,3} \cdot$ Pratibha Maurya $^{4} \cdot$ Rajeev Jain ${ }^{5} \cdot$ Saumya Nair $^{1} \cdot$ Rajendra K. Shukla $^{1}$. Sanjay Yadav ${ }^{1}$. Dhirendra Singh ${ }^{6} \cdot$ Vinay Kumar Khanna $^{1} \cdot$ Rajnish Kumar Chaturvedi $^{1} \cdot$ Mohana K. R. Mudiam $^{5}$. Rao Sethumadhavan ${ }^{2} \cdot$ Mohammad Imran Siddiqi $^{4} \cdot$ Devendra Parmar $^{1}$
\end{abstract}

Published online: 26 June 2019

C) Springer Science+Business Media, LLC, part of Springer Nature 2019

Correction to: Mol Neurobiol (2016) 53:3670-3689

https://doi.org/10.1007/s12035-015-9307-y

The original version of this article unfortunately contained an error at Fig. 10. The immunohistochemical data of GAD67, figures of prenatal and postnatal group alone were copied incorrectly during the preparation of figures.

The corrected Fig. 10 is hereby given below.

The online version of the original article can be found at https://doi.org/ 10.1007/s12035-015-9307-y

\author{
Devendra Parmar \\ parmardevendra@hotmail.com \\ Anshuman Singh \\ anshuman321_80@hotmail.com \\ Anubha Mudawal \\ anubha213@gmail.com \\ Pratibha Maurya \\ pratibha7728@gmail.com \\ Rajeev Jain \\ rajeevjain08@gmail.com \\ Saumya Nair \\ 88saumyanair@gmail.com \\ Rajendra K. Shukla \\ razshukla@gmail.com \\ Sanjay Yadav \\ sanjayitrc@gmail.com \\ Dhirendra Singh \\ drdhirendra@gmail.com \\ Vinay Kumar Khanna \\ vkkhanna1@gmail.com \\ Rajnish Kumar Chaturvedi \\ rajnish@iitr.res.in
}

\begin{abstract}
Mohana K. R. Mudiam
mohanitrc@gmail.com

Rao Sethumadhavan

rsethumadhavan@vit.ac.in

Mohammad Imran Siddiqi

mi_siddiqi@cdri.res.in
\end{abstract}

1 Developmental Toxicology Division, CSIR-Indian Institute of Toxicology Research, Post Box No. 80, M.G. Marg, Lucknow 226 001, Uttar Pradesh, India

2 School of Bio Sciences and Technology, Vellore Institute of Technology, Vellore 632014, Tamil Nadu, India

3 Academy of Scientific and Innovative Research (AcSIR), New Delhi 110025, India

4 Molecular and Structural Biology Division, CSIR-Central Drug Research Institute, Lucknow 226031, Uttar Pradesh, India

5 Analytical Chemistry Division, CSIR-Indian Institute of Toxicology Research, Post Box No. 80, M.G. Marg, Lucknow 226001, Uttar Pradesh, India

6 Animal Facility Division, CSIR-Indian Institute of Toxicology Research, Post Box No. 80, M.G. Marg, Lucknow 226001, Uttar Pradesh, India 

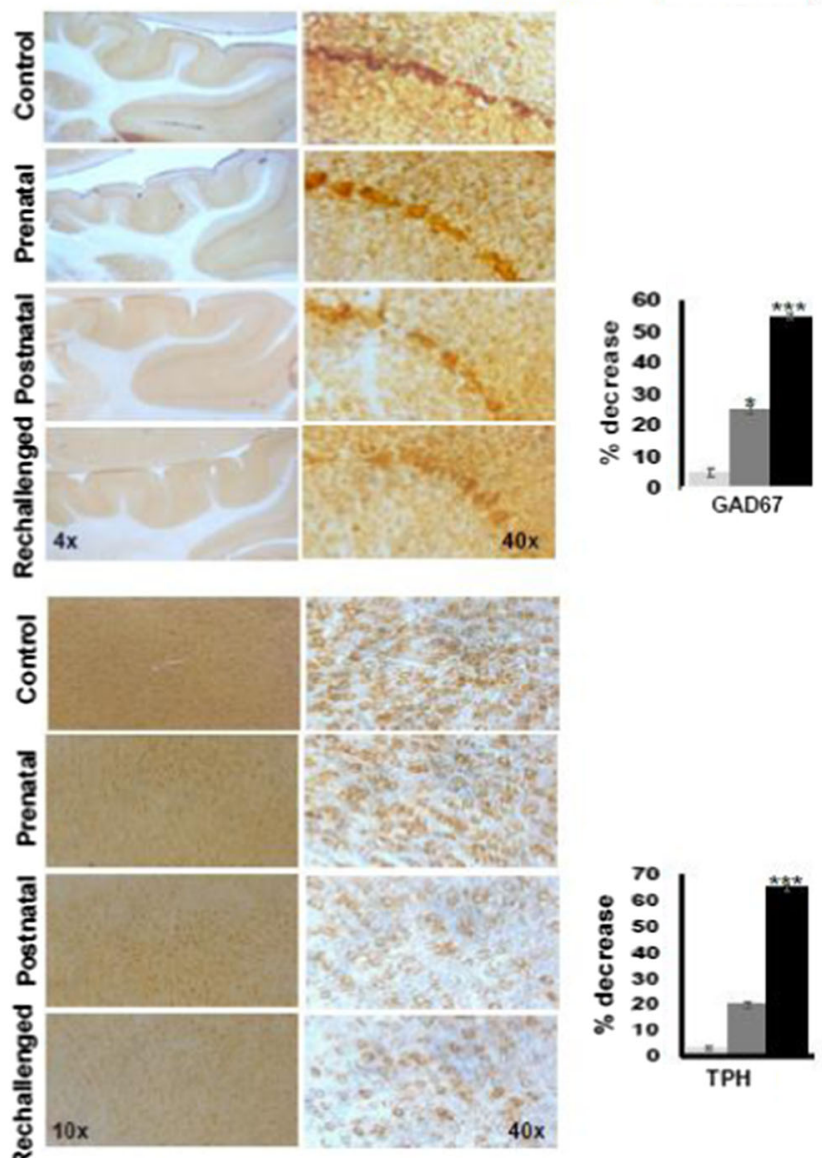

Fig. 10 Immunohistochemistry in brain regions of prenatally exposed offsprings which were rechallenged with cypermethrin at adulthood. Immunohistochemical analysis showing postnatal effect of cypermethrin on GAD67, ChAT, TPH, and TH in cerebellum, hippocampus, frontal cortex, and corpus striatum of the offsprings

This replacement of figure will not affect the total outcome of the paper.

Publisher's Note Springer Nature remains neutral with regard to jurisdictional claims in published maps and institutional affiliations.
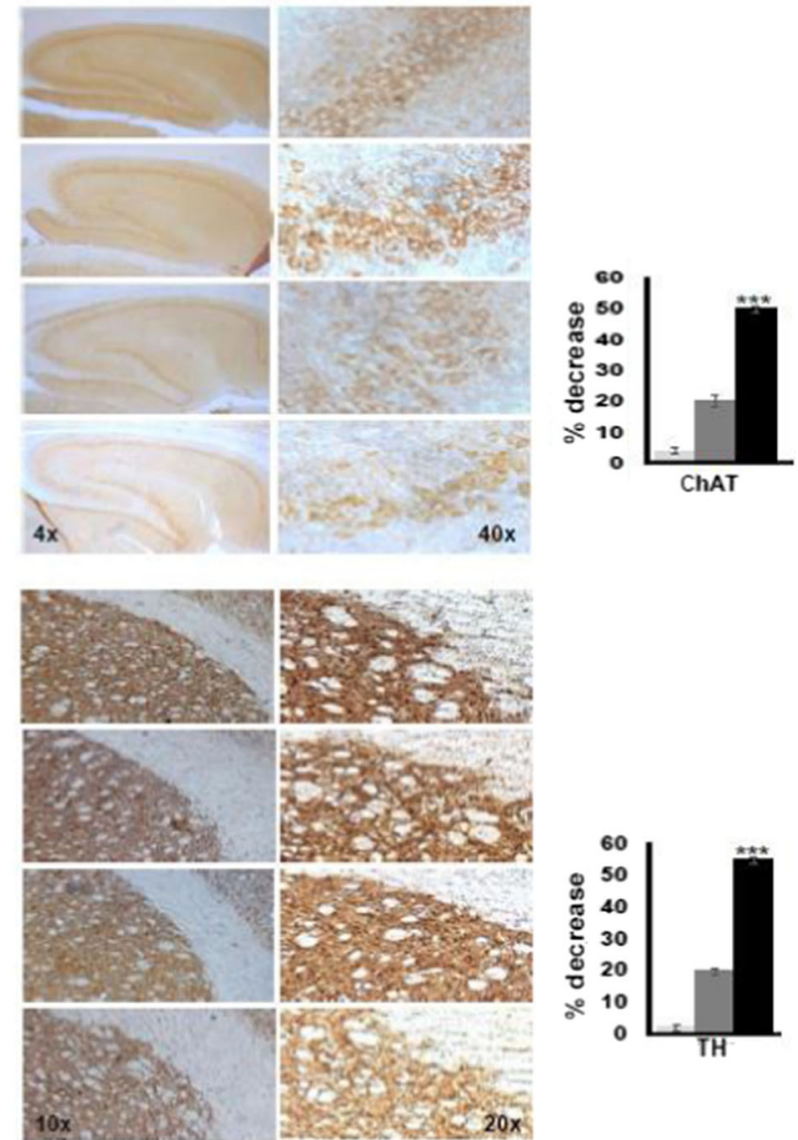

raised on control dams or dams treated with cypermethrin during gestation and subsequently treated orally with cypermethrin $(10 \mathrm{mg} / \mathrm{kg} \times 6$ days $)$ at adulthood (12 weeks) postnatally. Bar diagram represents quantitative analysis. (All the values represent mean \pm SEM of three experiments; $* P<0.05$; $* * P<0.01 ; * * * P<0.001)$ 